\title{
Effect of Oral Submucous Fibrosis on Jaw Dimensions
}

\author{
Aarati Panchbhai
}

Department of Oral Medicine and Radiology, Sharad Pawar Dental College and Hospital, Sawangi (M), Wardha, Maharastra, India

Cite this article as: Panchbhai A. Effect of Oral Submucous Fibrosis on Jaw Dimension. Turk J Orthod 2019; 32(2): 105-9.

\begin{abstract}
Objective: The disuse of the jaws owing to the restricted movement of the mandible in advanced cases of oral submucous fibrosis (OSMF) may have an effect on the morphologic features of the jaw bones. The purpose of the present study was to determine the jaw bone measurements in patients with OSMF and to compare the measurements in normal subjects and Caucasian norms.

Methods: The lateral cephalograms of 59 subjects ( 43 males and 16 females) with OSMF and 44 normal subjects ( 23 males and 21 females) in the age group of 18-45 years were collected. The jaw measurements were performed using Burstone analysis, and the relevant linear measurements of jaw sizes included were N-A, N-B, N-ANS, ANS-PNS, ANS-Gn, Ar-Go, and Pg-Go. The sex-wise comparison was performed using unpaired t-test, and measurements were compared with other studies using $Z$ test.

Results: In the present study, sex-wise comparison was found to be significant with greater jaw measurements in males than in females in patients with OSMF. Overall, the measurements were less or equal in patients with OSMF than in normal subjects except for $\mathrm{N}$-ANS and Pg-Go. When study measurements were compared with Burstone measurements, differences were significant with greater and less measurements.
\end{abstract}

Conclusion: Overall, the jaw measurements were less or equal in patients with OSMF than in normal subjects except for mandibular body length and middle third facial height that may need further evaluation.

Keywords: Oral submucous fibrosis, burstone analysis, lateral cephalograms, linear jaw measurements

\section{INTRODUCTION}

Oral submucous fibrosis (OSMF) is a chronic premalignant condition that affects various portions of the oral cavity, as well as the pharynx. The prevalence of OSMF is higher in southeast Asia, South Africa, and Middle East regions. OSMF causes progressive fibrosis of submucosal tissues and juxta-epithelial inflammatory reactions leading to stiffness of the oral mucosa. In severe cases, the mouth opening may become restricted partially or completely $(1,2)$.

The disuse of the jaws owing to the restricted movement of the mandible may have an effect on the morphologic and biomechanical features of the jaw bones, joints, and muscles (3-5). One of the factors that influence the form and structure of the bone is its mechanical loading. It is well documented that if a bone or muscle is not used for a large interval of time, then disuse atrophy may occur. Contrarily, hyperfunction or increased demand due to increased masticatory forces should lead to hypertrophy. The bone undergoes continuous formation and resorption to achieve its function. In the adult skeleton, the bony homeostasis is maintained when these two processes are in balance. Thus, the remodeling of the bone in response to mechanical loading on the bone can regulate bone resorption, and formation maintains its form/shape and bone mass (amount of bone). The significant restriction of jaw movement or immobilization may stimulate resorption and suppress bone formation (3-8).

In view of this, the present study was conducted in patients with OSMF to assess the amount or size of the maxillary and mandibular jaw bones with regard to their linear measurement to determine whether the reduction 
in jaw bone movements due to fibrosis has any influence on the size of the jaw bones. This may predict whether mandibular jaw hypomobility can be a forerunner of altered jaw dimensions in patients with OSMF.

Accordingly, the present study intended to determine the linear measurements of the maxilla and mandible in patients with OSMF with their sex-wise comparisons. In addition, the measurements in patients with OSMF were compared with the linear measurements of the maxilla and mandible in normal subjects and Caucasian norms.

\section{METHODS}

The study was approved by the ethics committee. The study was conducted in the Department of Oral Medicine and Radiology, Sharad Pawar Dental College and Hospital, DMIMS DU, Sawangi-M, Wardha, Maharashtra, India. The study participants were recruited from the outpatient department of the hospital. A total of 59 clinically diagnosed cases of patients with OSMF were included in the study based on their clinical findings and relevant habit history.

For measurements, the lateral cephalograms were obtained. The measurements were performed using Burstone hard tissue analysis of cephalometrics $(9,10)$ on skeletal profile. The lateral cephalometric radiographs were collected in a standardized manner in centric occlusion with the Frankfort horizontal plane (HP) oriented horizontally using a Planmeca ProLine CC machine (Windows Vista (R) SP1, 32 bit; Helsinki, Finland). The study intended to measure the maxillary and mandibular jaw sizes, and the linear measurements relevant to the study were measured. The measurements included in the study were N-A (linear), N-B (linear), N-ANS (linear), ANS-Gn (linear), ANS-PNS (linear), Ar-Go (linear), and Pg-Go (lin-

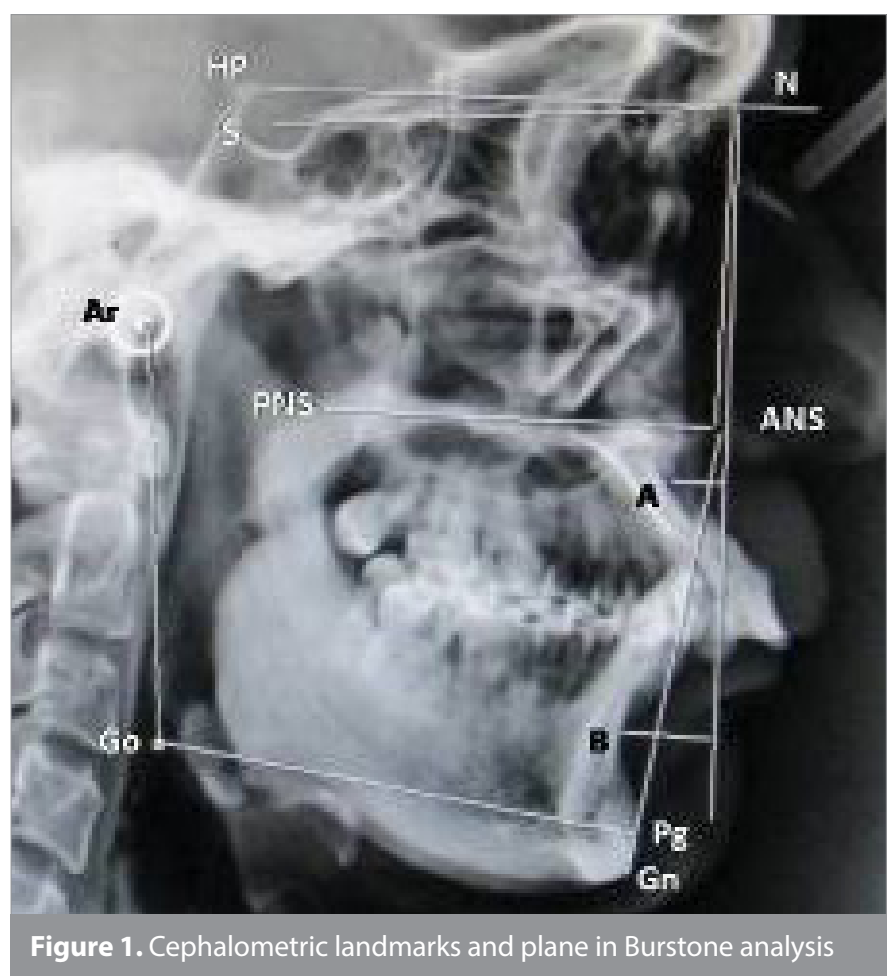

ear). The baseline used in Burstone analysis is the HP constructed by drawing a line intersecting at $N 7^{\circ}$ from the Sella-Nasion plane; the measurements in this analysis are made either parallel to or perpendicular to this plane. The various included landmarks (Figures 1,2$)$ relevant to the study were (10-14):

- $\quad$ Nasion (N) - the most anterior point of the nasofrontal suture in the midsagittal plane,

- Anterior nasal spine (ANS) - the anterior-most midsagittal point on the tip of the sharp bony process of the maxilla,

- Subspinale (A)-the deepest midsagittal point on the concavity between ANS and prosthion,

- Supramentale (B) - the deepest point in the midsagittal plane on the concavity between infradentale and pogonion,

- Posterior nasal spine (PNS) - the most posterior point on the contour of the palate,

- Articulare (Ar) - the intersection of the sphenoid and the posterior border of the condyle,

- Gonion (Go) - constructed by bisecting the posterior ramal plane and mandibular plane,

- Gnathion (Gn) - constructed by bisecting the facial plane and tangent to the lower border of the mandible.

$\mathrm{N}-\mathrm{A}$ measurement describes the position of the apical base of the maxilla in relation to nasion. To measure this, perpendicular to the HP is dropped from $\mathrm{N}$ ( $\mathrm{N}$ perpendicular), and horizontal distance parallel to the HP is measured from point $A$.

N-B measurement describes the position of the apical base of the mandible in relation to nasion. It is obtained by measuring the distance between point $\mathrm{B}$ and nasion perpendicular ( $\mathrm{N}$ perpendicular) parallel to the HP.

$\mathrm{N}$-ANS measurement describes the distance between $\mathrm{N}$ and ANS measured perpendicular to the HP providing the middle third facial height.

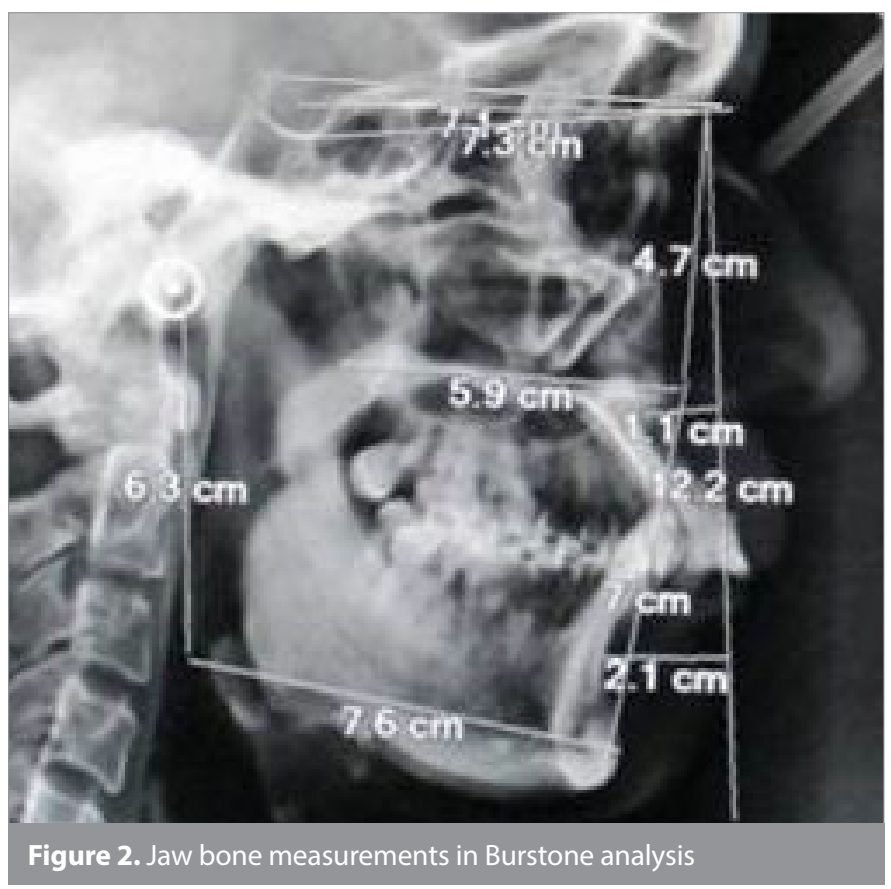


ANS-Gn measurement describes the distance between ANS and Gn measured perpendicular to the HP providing the lower third facial height.

ANS-PNS measurement describes the distance between these two points measuring the total effective maxillary length.

Ar-Go measurement describes the linear distance between articulare and gonion providing the mandibular ramal length.

Pg-Go measurement describes the linear distance between gonion and pogonion providing the mandibular body length.

The measurements were recorded in millimeters. The recorded measurements were tabulated and subjected for statistical analysis.

Statistical analysis was performed using Statistical Package for Social Sciences version 21.0 (IBM Corp.; Armonk, NY, USA). Z test was used for comparison between the studies. Student's unpaired t-test was used for sex-wise comparison. A P (probability) value $\leq 0.05$ was considered as statistically significant.

\section{RESULTS}

In the present study, sex-wise comparison was found to be significant (P values: N-A: 0.001, N-B: 0.009, ANS-Gn: 0.003, ANS-PNS: 0.002, Pg-Go: 0.01, and Ar-Go: 0.0001) in patients with OSMF for all the measurements except for $\mathrm{N}$-ANS. The measurements were greater in OSMF males than in OSMF females (Table 1).

When the measurements in patients with OSMF were compared with normal subjects, there were significant differences in measurements for N-ANS (significant $Z$ values: 5.76 and 2.67), ANS-Gn (significant $Z$ values: 7.31 and 5.28), Ar-Go (significant $Z$ values: 10.1 and 9.18), and Pg-Go (significant $Z$ values: 0.31 and 12.32 ) in both males and females, respectively. When the measurements were compared, the values were less in OSMF females than in normal females except for Pg-Go measurements. Overall, OSMF males had less measurement than normal males except for N-ANS and Pg-Go (Table 2).

When the measurements in patients with OSMF were compared with Burstone measurements in the Caucasian population, differences in values were significant (significant $Z$ values: N-A: 5.89

\begin{tabular}{|c|c|c|c|c|c|c|c|}
\hline \multicolumn{8}{|c|}{ Student's unpaired t-test } \\
\hline & Sex & $\mathbf{N}$ & Mean & Std. deviation & Std. error of the mean & t-Value & $\mathbf{p}$ \\
\hline N-A & Female & 16 & 0.9 & 1.01 & 0.10898 & & \\
\hline N-B & Male & 43 & 5.1 & 4.91 & 0.16707 & 2.68 & $0.009, \mathrm{~S}$ \\
\hline N-ANS & Female & 16 & 54.3 & 1.69601 & 0.42400 & & \\
\hline \multirow[t]{2}{*}{ ANS-Gn } & Male & 43 & 66.6 & 0.53006 & 0.08083 & 3.08 & $0.003, \mathrm{~S}$ \\
\hline & Female & 16 & 61.4 & 0.66448 & 0.16612 & & \\
\hline ANS-PNS & Male & 43 & 54.1 & 0.44262 & 0.06750 & 3.27 & $0.002, \mathrm{~S}$ \\
\hline \multirow[t]{2}{*}{ Ar-Go } & Male & 43 & 56.0 & 0.49923 & 0.07613 & 4.18 & $0.0001, \mathrm{~S}$ \\
\hline & Female & 16 & 48.4 & 0.85851 & 0.21463 & & \\
\hline
\end{tabular}

Table 2. Comparisons of mean values of linear jaw measurements between patients with OSMF and normal subjects

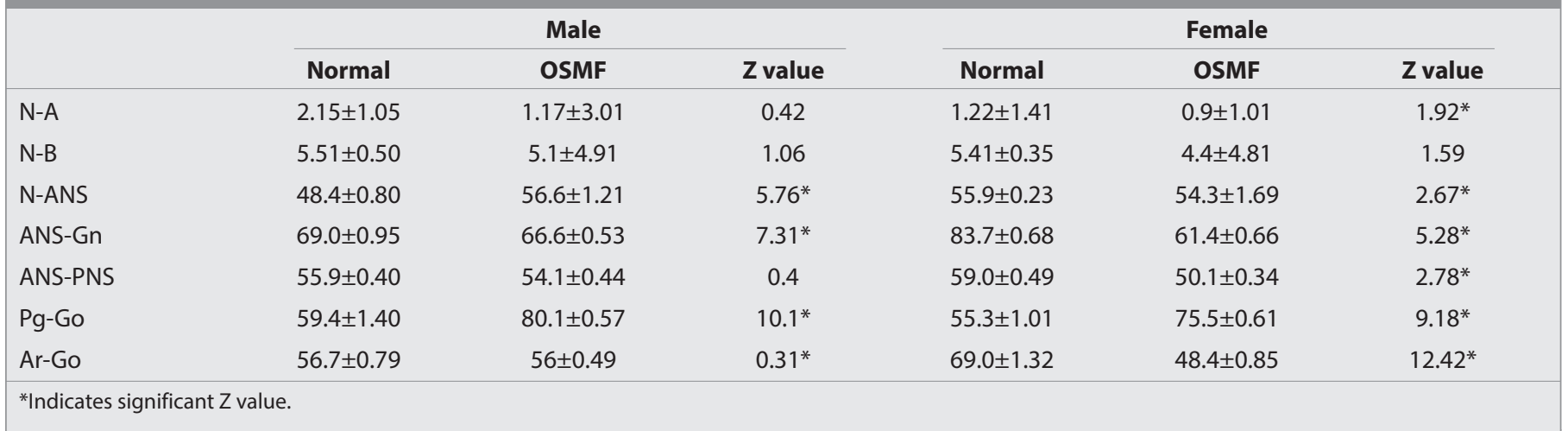


Table 3. Comparisons of mean values of linear jaw measurements between the present study and Burstone measurements

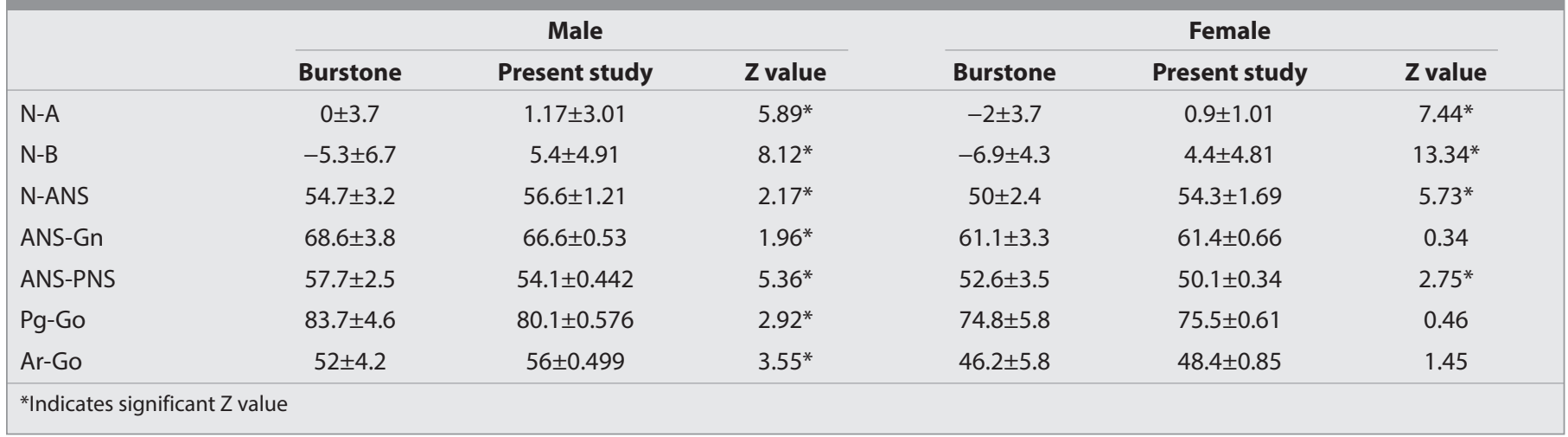

and 7.44, N-B: 8.12 and 13.34, N-ANS: 2.17 and 5.73, and ANSPNS: 5.36 and 2.75) in males and females except for ANS-Gn, Ar$\mathrm{Go}$, and $\mathrm{Pg}-\mathrm{Go}$ in females. The values in patients with OSMF were greater for N-A, N-B, N-ANS, and Ar-Go and less for ANS-Gn, ANSPNS, and Pg-Go than those in the Caucasian population in both males and females (Table 3 ).

\section{DISCUSSION}

The present study assessed the linear measurements of the jaw bones (maxilla and mandible) to determine jaw bone sizes in patients with OSMF. The study conducted sex-wise comparisons and comparisons with measurements in normal subjects and Burstone study. In the present study, the measurements were significantly higher in males than in females in patients with OSMF, suggesting greater dimensions of the jaw bone in males than in females.

Overall, the measurements were equal or less in patients with OSMF than in normal subjects except for N-ANS and Pg-Go (Table 2). Thus, the middle third facial height and mandibular body length were greater in patients with OSMF than in normal subjects. The total effective maxillary length and lower third facial height were smaller, and mandibular ramal length was more or less equal in OSMF males than in normal males. The middle third facial height, total effective maxillary length, lower third facial height, and mandibular ramal length were smaller in OSMF females than in normal females.

There was a significant difference between Burstone measurements in the Caucasian population and present study measurements in patients with OSMF except for ANS-Gn, Ar-Go, and $\mathrm{Pg}-\mathrm{Go}$ in females. The values in patients with OSMF for measurements were greater for N-A, N-B, N-ANS, and Ar-Go and less for ANS-Gn, ANS-PNS, and Pg-Go than those in the Caucasian population in both males and females. Thus, the comparisons revealed the variable positions of the apical base of the maxilla and mandible in relation to nasion, greater middle third facial height, and ramal length, whereas less lower facial height and total effective maxillary and mandibular length in the Caucasian population (10) (Table 3).

India is one of the most populous countries with regional variations, and demographic and sociocultural characteristics of the communities are too complex and thus incomparable. The normal measurements of a particular locality may not be considered normal for other regions. Hence, the present study also included comparisons of measurements in patients with OSMF with jaw measurements in the normal population simultaneously, and it was observed that the overall measurements were either relatively equal or less than the normal population.

The structure and amount of the bone are determined by genetic blueprint and by various regulatory factors. The ability of the bone to alter its structure and to adapt to mechanical loads entails that mechanical forces can regulate bone turnover or remodeling; increased loads should increase formation, and unloading should have the opposite effect. The action of these factors, hormones, and cytokines on osteoclasts was proposed to be mediated by osteoblast-lineage cells, which possess the cognate receptors intimately linking osteoblast-osteoclast interaction to bone turnover (3-5). It may be anticipated that the restricted jaw movements or immobilization of the jaws in patients with OSMF may stimulate resorption and suppress formation. Although multiple factors probably may be involved in the maintenance of bone homeostasis, the active factor is seen presently in the form of restricted jaw bone movements in patients with OSMF.

The study was conducted to assess the influence of restricted jaw movements on the maxillary and mandibular jaw sizes. The findings in the present study may provide the baseline data for future studies. The variable observations when the jaw measurements in patients with OSMF were compared with normal subjects and the Caucasian population recommend further studies using broader sample size correlating with interincisal width and history of reduced mouth opening, i.e., the degree and years of partial or complete immobilization of the jaws, to reach a definite conclusion.

\section{CONCLUSION}

- In patients with OSMF, the measurements were significantly greater in males than in females

- Overall, the measurements were equal or less in patients with OSMF than in normal subjects except for middle third facial height and mandibular body length.

- The comparisons between Burstone measurements and present study measurements in OSMF revealed greater middle third facial height and ramal length, whereas less lower 
facial height and total effective maxillary and mandibular length in the Caucasian population.

Ethics Committee Approval: Ethics committee approval was received for this study from the Ethics Committee of Sharad Pawar Dental College and Hospital.

Informed Consent: Written informed consent was obtained from the patients who participated in this study.

Peer-review: Externally peer-reviewed.

Conflict of Interest: The author has no conflict of interest to declare.

Financial Disclosure: The author declared that this study has received no financial support.

\section{REFERENCES}

1. Wollina U, Verma S, Ali F, Patil K. Oral submucous fibrosis: an update. Clin Cosmet Investig Dermatol 2015; 8: 193-204. [CrossRef]

2. Das M, Manjunath C, Srivastava A, Malavika J, Musareth V. Epidemiology of Oral Submucous Fibrosis. Int J Oral Health Medical Res 2017; 3: 126-9.

3. Masthan K, Babu A, Shankari L, K Gaytri. Oral Submucous Fibrosis: A forerunner ofTMJ changes. Bisci Biotech Res Asia 2013; 10: 261-5. [CrossRef]

4. Rodan GA. Bone homeostasis. Proc Natl Acad Sci USA 1998; 10: 13361-2. [CrossRef]
5. Howard A, Steven B. Important ant role of motion in rehabilitation of patient with mandibular hypomobility: a review of literature. J Craniomandibular practice 1997; 5: 74-83 [CrossRef]

6. Skerry T M, Bitensky L, Chayen J, Lanyon LE. Early strain-related changes in enzyme activity in osteocytes following bone loading in vivo. J Bone Miner Res 1989; 4: 783-8. [CrossRef]

7. Frost H M. Intermediary Organization of the Skeleton. Boca Raton, FL: CRC; 1986. Metab Bone Dis Relat Res 1983; 4: 281-90

8. Baron R, Vignery A, Horowitz M. In: Bone and Mineral Research. Peck W A, editor. New York: Elsevier; 1984. pp. 175-243.

9. Trivedi K, Singh S, Shivamurthy DM, Doshi J, Shyagali T, Patel B. Analysis of cephalometrics for orthognathic surgery: Determination of norms applicable to Rajasthani population. Natl J Maxillofac Surg 2010; 1: 102-7.[CrossRef]

10. Burstone CJ, James RB, Legan H, Murphy GA, Norton LA. Cephalometrics for orthognathic surgery. J Oral Surg 1978; 36: 269-77.

11. Soni A, Alladwar N, Goel S, Chopra R, Sharma S. Evaluation of the Lateral cephalometric norms for Burstone analysis in Chhattisgarh by using Nemoceph software with Lateral cephalogram taken in natural head position. Int J O Health Dent 2015; 1: 114-9.

12. Rao S, Singaraju G, Mandava P, Ganugapanta V. Cephalometric analysis for orthognathic surgery. Annals and Essences of dentistry. 2015, Vol. VII-3; 1-10

13. Nanda R, Nanda RS. Cephalometric study of the dentofacial complex of North Indians. Angle Orthod 1969; 39: 22-8.

14. Grewal H, Sidhu SS, Kharbanda OP. Cephalometric appraisal of the craniofacial pattern in Indo-Aryans. J Indian Orthod 1995; 26: 438. 